\title{
Implementación de Entornos Personales de Aprendizaje para fortalecer las habilidades comunicativas
}

\author{
Implementation of Personal Learning Environments \\ to Strengthen Communication Skills
}

\author{
Implementação de entornos pessoais de aprendizagem \\ para fortalecer as habilidades comunicativas
}

\author{
Isabel Jiménez-Becerra ${ }^{1}$ \\ Luz Andrea Salamanca-Espinosa ${ }^{2}$ \\ Luis Guillermo López-López ${ }^{3}$
}

\section{Resumen}

El presente artículo pretende determinar la influencia del mismo en los procesos de aprendizaje en la asignatura de inglés en Básica Primaria. Parte de un recorrido teórico que evidencia algunas prácticas y aportes en el contexto educativo, luego demuestra una experiencia aplicada con un grupo de estudiantes entre los siete y nueve años de edad con el fin de corroborar sus alcances. Desde el marco metodológico, se implementa un diseño de investigación aplicada por medio de la Investigación-Acción-Participación (IAP). El análisis de datos cualitativos se realizó a partir de la técnica de observación participante y los datos cuantitativos a partir de la implementación de un encuesta que permitió identificar el avance en los procesos de aprendizaje centrado en las habilidades comunicativas. La implementación de la experiencia se generó por medio de un ambiente que concentró experiencias de aprendizaje individuales y colaborativas, a través de talleres, contenidos y recursos para la producción textual, gráfica y audiovisual. De este modo, se da sentido a la información que se recibe, lo que se construye desde los propios intereses, lo que se aprende y cómo cada individuo se hace visible a los otros en el mundo virtual y presencial. Se evidencia así, la importancia de los pLE (Entorno Personal de Aprendizaje) en la educación y cómo cada individuo es capaz de utilizar diversa información para la producción de conocimiento, el fortalecimiento de habilidades comunicativas y tecnológicas e impactar en otras dimensiones del ser humano, donde la tecnología es mediadora de espacios de interacción.

Palabras clave

PLE; habilidades comunicativas; información; enseñanza

1 Estudiante del Doctorado en Educación en la línea de Didácticas de la Lengua y la Literatura de las Ciencias Sociales de la Universidad Autónoma de Barcelona, España. Profesora investigadora perteneciente al grupo de investigación Proventus del Centro de Tecnologías para la Academia de esta Universidad de la Sabana. Lidera el proyecto profesoral Didácticas Innovadoras mediadas por tic. Correo electrónico: isabel.jimenez@ unisabana.edu.co

2 Magíster en Proyectos Educativos Mediados por tic de la Universidad de la Sabana (Chía, Cundinamarca-Colombia). Licenciada en Pedagogía Infantil de la Universidad Distrital Francisco José de Caldas. Maestra de básica primaria de la sed Bogotá. Coinvestigadora principal del proyecto de investigación "Implementación de entornos personales de aprendizaje para fortalecer las habilidades comunicativas en estudiantes de tercero de primaria del colegio José Jaime Rojas". Correo electrónico: luzsaes@unisabana.edu.co

3 Magíster en Proyectos Educativos Mediados por tıc de la Universidad de la Sabana. Maestro de básica secundaria de la sed Bogotá. Coinvestigador del proyecto "Didácticas innovadoras mediadas por tIC". Investigador del grupo de investigación Proventus del Centro de Tecnologías para la Academia de la Universidad de la Sabana (Chía, Cundinamarca-Colombia). Correo electrónico: luisloplo@unisabana.edu.co 


\section{Abstract}

This paper aims to determine its influence in the learning processes in English in Basic Elementary school. It starts with a review of theory that shows some practices and contributions in the educational context, followed by the demonstration of an experience applied to a group of students between the ages of seven and nine, in order to corroborate their range. Based on the methodological framework, an applied research design is implemented through participatory action research (PAR). The analysis of qualitative data was performed using the participant observation technique, and the quantitative data were analyzed based on the implementation of a survey that made it possible to identify the progress made in the learning processes focused on communication skills. The experience was implemented through an environment that concentrated individual and collaborative learning experiences by means of workshops, content and resources for textual, graphic and audiovisual production. Thus, it gives meaning to the information received, what is built from one's own interests, what is learned and how each individual becomes visible to others both in the virtual and face-to-face world. Thus, the importance of PLE (Personal Learning Environment) in education becomes evident, as well as how each person is capable of using different information to produce knowledge, strengthen communicative and technological skills, and impact other dimensions of the human being, where technology is a mediator of interaction spaces.

\section{Keywords}

PLE; communication skills; information; teaching

\section{Resumo}

0 presente artigo visa determinar sua influência nos processos de aprendizagem de inglês no ensino básico. Inicia com um percurso teórico que evidencia algumas práticas e contribuições no contexto educativo, despois demonstra uma experiência aplicada com um grupo de estudantes entre os sete e os nove anos de idade com o fim de corroborar suas conquistas. Desde o quadro metodológico, apresenta-se um projeto de pesquisa aplicado por meio da Investigação-Ação-Participação (IAP). A análise de dados qualitativos foi realizada a partir da observação participante e os dados quantitativos com a implementação de uma enquete que permitiu identificar 0 avanço nos processos de aprendizagem centrada nas habilidades comunicativas. A implementação da experiência foi gerada por meio de um ambiente que coletou experiências de aprendizagem individuais e colaborativas, através de oficinas, conteúdos e recursos para a produção textual, gráfica e audiovisual. Dessa forma, a informação coletada adquire sentido, o que constitui desde interesses próprios o que é aprendido e como cada indivíduo se faz visível aos outros no mundo virtual e presencial. Evidencia-se, assim, a importância dos PLE (Entorno Pessoal de Aprendizagem) na educação e como cada indivíduo é capaz de utilizar diversas informações para a produção de conhecimento, o fortalecimento de habilidades comunicativas e tecnológicas e impactar em outras dimensões do ser humano, onde a tecnologia é mediadora de espaços de interação.

\section{Palavras chave}

PLE; habilidades comunicativas; informação; ensino

\section{Artículo recibido el 8 de marzo de 2017 y aprobado el 28 de julio de 2017}

\section{Introducción}

En el contexto educativo se han aplicado los entornos personales de aprendizaje (PLE por sus siglas en inglés) como parte del proceso de enseñanzaaprendizaje. Según López (2015) -quien abordó parte de este rastreo en el proyecto de investigación Didácticas Innovadoras Mediadas por TIC del grupo de investigación Proventus del Centro de Tecnologías para la Academia de la Universidad de la Sabana-, en el contexto latinoamericano se destacan países como España, México, Ecuador, Venezuela y Colombia. Dentro de su ejercicio recopiló algunas de las experiencias que forman parte de este marco.

En primer lugar, se encuentra Gil (2012), de la Universidad de Burgos, quien presenta un completo estudio de un caso que se centra en la forma como la creación y utilización de PLE y el fortalecimiento del uso de las TIC mejoran el proceso de enseñanza- aprendizaje. Esta experiencia se llevó a cabo en el contexto de un centro educativo, con estudiantes de Lengua Castellana. La investigación se estructura en dos partes: la primera, aborda la conceptualización de los aspectos relacionados con internet, la web 2.0 y las metodologías educativas desarrolladas para su implementación; en la segunda, se presenta la investigación desarrollada profundizando en la utilización de las tecnologías en el ambiente educativo y usando blogs y redes sociales como elementos fundamentales en la creación de los PLE. Gracias a estas estrategias, ha mejorado el aprendizaje digital, tanto de los alumnos como del docente.

En la revista e-Co, Lara y Moraga (2013), se presenta una investigación práctica que consiste en el diseño de un PLE aplicable a un aula de secundaria, específicamente al área de Ciencias Sociales. Se introducen novedosos planteamientos desde una 
doble perspectiva: por un lado, el docente que está en contacto diario con la realidad del aula, afrontando las posibilidades y los problemas en la aplicación de estos nuevos enfoques; $y$, por el otro, la formación del profesorado para presentar las propuestas aplicadas a Ciencias Sociales, Geografía e Historia.

Por otro lado, en su artículo "Una experiencia de creación de un entorno personal de aprendizaje. Proyecto Dipro 2.0", Rodríguez (2013), habla del proyecto Dipro 2.0, como una de las pocas experiencias que se han desarrollado respecto de la creación de un Entorno Personal de Aprendizaje (PLE). En el artículo se describen los objetivos, la secuencia seguida para la implementación del proyecto y unas valoraciones finales sobre su estado, y cómo sus resultados apuntan a poder desarrollar acciones de formación y capacitación del profesorado universitario en TIC, y de qué manera el entorno telemático producido supera la visión tradicional de los PLE, en un enfrentamiento con los Learning Management System (LMS), y permite incorporar de manera unificada los dos componentes en acciones formativas institucionales.

En un artículo de Ruiz-Palmero, Sánchez Rodríguez y Gómez García (2013), basado en un estudio realizado con el objetivo de conocer el papel que los PLE desempeñan en la formación del alumnado de la Facultad de Ciencias de la Educación de la Universidad de Málaga, se han estudiado cuáles son las aplicaciones y herramientas que más emplea el alumnado en su aprendizaje no formal. Dentro de estas predomina el uso de las redes sociales, como el Facebook, y concluyen que en general el alumnado no utiliza efectivamente los medios de que dispone con fines académicos.

Marín, Bennasar y Pérez (2014) contemplan la creación y el mantenimiento de entornos y redes personales de aprendizaje y su integración en entornos virtuales institucionales de aprendizaje (EVA) como estrategias que facilitan y promueven el aprendizaje colaborativo. Desde una visión educativa, los resultados de esta experiencia indican que los alumnos construyen sus PLE y PLN con base en los nuevos conocimientos adquiridos.
Santamaría (2010) expone en su artículo "Evolución y desarrollo de un entorno personal de aprendizaje en la Universidad de León" cómo, en la Facultad de Educación de dicha universidad, se desarrolla desde 2016 un trabajo con estudiantes de Las Matemáticas y su Didáctica, que los compromete con una metodología participativa, de trabajo colaborativo, guiada por la evaluación continua en función formativa. Desde hace más de cuatro años se han implementado distintas herramientas basadas en la web, para llegar a lo que hoy se llama un entorno personal de aprendizaje (PLE).

Llorente (2012) presentan un marco teórico en el cual se expone la evolución de los diferentes momentos en el desarrollo de internet y las posibilidades educativas, a través de la evolución del aprendizaje virtual (e-learning), el análisis crítico de los errores en su aplicación, y la evolución hacia el concepto de aprendizaje virtual 2.0, a través de herramientas como las redes sociales y los PLE, sus características más significativas, así como el potencial educativo en las instituciones de enseñanza superior.

En el artículo "Entornos Personales de Aprendizaje: ¿final o futuro de los Eva?", Ampudia y Lourdes (2012) sostienen que en la actualidad ha surgido una nueva discusión que cuestiona el papel de las aplicaciones EVA y su futuro ante la web 3.0 y el uso masivo de redes sociales. La reflexión se centra en cómo producir contenidos, integrar comunidades virtuales, superar la incompatibilidad tecnológica y las desventajas de la administración centralizada en línea. Ante este panorama surgen los PLE. Se pretende analizar la pertinencia de esta perspectiva en la UAM-Xochimilco.

Cabero (2013), en "Educación y cultura en la sociedad de información", presenta un planteamiento acerca de cómo las comunidades virtuales (cv), los PLE, los productos de la web 2.0, la computación en la nube y los medios sociales están impactando en el terreno educativo y están llevando a los estudiantes a desempeñar un papel más activo en el proceso de aprendizaje y a integrar en su formación no solo los contextos formales, sino también los informales y no formales. 
Dentro de las experiencias encontradas en el medio colombiano, aplicadas en instituciones de educación distrital, están las tesis de Restrepo (2016) y Bohada (2016), implementó estrategias metodológicas que integran las estrategias de lectura a las herramientas que utilizan los estudiantes dentro de sus PLE, y demostró cómo se integran las estrategias de lectura a los PLE, con el uso adecuado de las herramientas de internet para potenciar, afianzar y facilitar la comprensión de textos, que a su vez favorece la adquisición de nueva información en cualquier área del conocimiento, no solo para mejorar la forma de leer sino también de escribir.

Señala Bohada (2016) que un proceso similar al anterior se implementó en el Colegio Tomas Carrasquilla en Bogotá con estudiantes del grado quinto de primaria en el área de Tecnología e Informática. Se diseñó e implementó un ambiente de aprendizaje inscrito en el marco de la robótica pedagógica y el software social, herramientas que favorecen los procesos de enseñanza y aprendizaje en la educación. Se obtuvo como resultado un ambiente de aprendizaje mediado por software social, cuya implementación favoreció en los estudiantes la apropiación de los conceptos básicos de la robótica, así como el desarrollo de habilidades del pensamiento tales como recordar, aplicar, comprender, analizar, evaluar y crear a través de la interacción y el manejo de herramientas de la web y la construcción del PLE.

De acuerdo con lo planteado en el presente estudio acerca de los Ple se observa que, a pesar de ser un recurso de origen reciente, diversos autores han participado en su conceptualización, dándole una marcada importancia como recurso para cambiar y mejorar la forma en que las personas aprenden.

Su aplicación se ha enfatizado en la utilización de las nuevas tecnologías de la información y la comunicación TIC, donde se involucran herramientas web, uso de redes sociales entre otras redes de aprendizaje, que están disponibles en la red. El incremento de los estudios que lo aplican se ha hecho evidente en el contexto educativo por ser un enfoque que emplea recursos TIC que están en constante evolución y desarrollo.
Los PLE tienen auge en el medio educativo colombiano, pues las instituciones educativas están mejorando su infraestructura tecnológica y están facilitando la formación docente mediante el aprendizaje de este nuevo enfoque. Se busca que los maestros involucren sus conocimientos en la práctica educativa, y que, en este proceso, se mejoren el rendimiento y la motivación de los estudiantes.

\section{Antecedentes}

Según Navas (2013), el término Personal Learning Environments aparece por vez primera en el año 2001 con una primera cita en un artículo de Olivier y Liber titulado "Lifelong learning: The need for portable personal learning environments and supporting interoperability standards". En él, dichos autores expresan la necesidad de una plataforma universal que almacene registros de aprendizaje para poder accederlos con y sin conexión. Como ejemplo de una concreción de su idea, Olivier y Lieber presentan Colloquia. Se trata de un sistema considerado como la primera implementación de PLE, desarrollado por la University of Wales, Bangor (Reino Unido), que soporta el aprendizaje activo y basado en proyectos, en el que cada usuario puede actuar a la vez como creador y consumidor de la información. Los alumnos pueden compartir recursos con sus colegas, invitar a los amigos a participar en actividades, enviar mensajes privados, etc.

Posteriormente, en el año 2004, en Gran Bretaña, se empleó oficialmente el concepto de PLE en un congreso realizado por el Joint Information System Committee, que trató de entornos centrados en el estudiante y que expuso, además, la necesidad de desarrollar plataformas personalizadas para la gestión de su aprendizaje. Se pretendía con ello crear un modelo estandarizado para los PLE, de tal forma que se configuraran en un software y de esta manera implantarlo como tecnología.

Hacia el año 2007, se reconoció que los PLE no son una aplicación de software, sino más bien un nuevo enfoque sobre el uso de las tecnologías como medio de apoyo para el aprendizaje. Atwell (2007) señaló que "es más bien un tema educativo, debido a 
que la evolución y avance en las tecnologías inciden para los cambios en educación".

La importancia que han venido tomando los PLE se pone de manifiesto con la realización de una serie de actividades entre las que destaca la PLE Conference (conferencia sobre los PLE), constituida como un espacio para la colaboración científica y el intercambio de ideas donde intervienen autores de varios países, como Reino Unido, España, Alemania, Portugal, con la finalidad de describir el concepto y contribuir a su evolución. Se han realizado cinco ediciones periódicas: en 2010 en Barcelona, España; en 2011 en Southampton, Reino Unido; en 2012 en Aveiro, Portugal y Melbourne, Australia; en 2013 en Berlín, Alemania y Melbourne, Australia y en 2014 en Tallin, Estonia.

En el año 2010, durante la celebración de la primera PLE Conference, se propuso la creación de un foro periódico dedicado exclusivamente a este tema, donde se tratara el asunto de la tecnología educativa como eje desde donde podrían abordarse otros temas. En las tres primeras ediciones de este congreso existió una evolución en el tratamiento del concepto. En la primera, la discusión se centró sobre si era un concepto tecnológico o pedagógico.

En el año 2011, en la segunda edición del Congreso, se analizó la evolución tecnológica que facilitaba la gestión de los PLE. También se habló sobre las competencias de los aprendices y docentes comprometidos con los PLE así como su participación en cursos formales y no formales.

En la edición de 2012, se mostraba ya un consenso acerca del concepto, lo que permitió que el eje de la discusión se centrara en el análisis de los PLE en contextos formales e informales, además del uso de servicios en red para su ejecución.

Desde su primera edición, la PLE Conference ha sido primordial para la actividad científica en torno a este concepto. Las ponencias presentadas en el congreso pueden considerarse como parte de su evolución y desarrollo; un total de 321 autores de 32 países con una producción científica de 196 artículos, $98,46 \%$ en inglés y $1,54 \%$ en español (Fernández, 2016, p. 9).
En cuanto a las tendencias y los estudios sobre los PLE, Gallego y Chávez (2014) presentan alguna información donde muestran datos estadísticos de investigaciones y publicaciones encontradas en el periodo comprendido entre 2012 y 2014, señalando que se han revisado un total de 376 registros con información sobre PLE, incluyendo artículos de revistas, comunicaciones en eventos científicos, capítulos de libros y tesis doctorales. Se han elegido aquellos que son investigaciones empíricas referidas explícitamente a los PLE publicados desde 2009, lo cual significa un total de 65 trabajos. En estas estadísticas se muestra que aproximadamente la mitad de los trabajos analizados (49\%) han sido publicados en revistas científicas, la mayoría de los cuales (34\%) corresponden al periodo entre 2013 y abril de 2016. Se destaca que un $44 \%$ de los trabajos han sido presentados en el PLE Conference; $38 \%$ han sido tomados de las memorias de este congreso y un $6 \%$ de eLearning Papers1. Además, cuatro de los cinco capítulos pertenecen a un mismo libro y existe una presencia muy escasa de tesis en el tema.

No se han encontrado trabajos empíricos sobre PLE en el año 2009. En 2012 se observan 17 en total, 16 de ellos escritos en inglés y solo uno en español. En 2013 se observan 22 en total, 15 en inglés y 7 en español. De hecho, es clara la importancia de la publicación en lengua inglesa para la investigación empírica sobre PLE, si se compara con la de español, ya que un $81,5 \%$ de los trabajos son en esta lengua. Sin embargo, casi la mitad de los presentados en 2013 son en español; y ese mismo año se presentaron 15 trabajos en inglés, en comparación con 16 del año inmediatamente anterior.

La importancia que ha tomado el concepto de los PLE como enfoque para el aprendizaje con TIC se hace evidente con la existencia de una amplia variedad de literatura basada en propuestas y experiencias. Se encuentran trabajos como el de Noguera, García y Gros (2014), Just4me: Diseño pedagógico y funcional de un PLE para la autogestión del aprendizaje en distintos contextos, quienes describen la metodología utilizada para fundamentar un entorno que integra herramientas y funcionalidades, diseñado con el 
fin de apoyar a los profesionales a dirigir su propio proceso de aprendizaje a lo largo de la vida, fundamentados exclusivamente en las características de las herramientas y recursos tecnológicos que componen el entorno personal. Otros, en cambio, enfatizan los aspectos psicopedagógicos que se derivan de la primera parte del aprendizaje personal, y que se centran en cómo ayudar a los estudiantes a crear y gestionar este tipo de entornos como un instrumento de aprendizaje.

Más allá de estos dos aspectos tecnológicos, o los psicopedagógicos, existen dos elementos o rasgos compartidos que son de especial interés para el ámbito educativo. Por una parte, Coll y Engel (2014) destacan la necesidad de actualización a lo largo de toda la vida para ir adaptándose a los cambios que conlleva la era digital. Por otra, la extensión de los procesos de aprendizaje de las instituciones de educación formal al conjunto de contextos o sistemas de actividad como la familia, el trabajo, las instituciones, etc., que proporcionan oportunidades para aprender. En el fundamento de los PLE, también se encuentra una visión del aprendiz como persona activa que busca, crea, adapta y comparte contenidos; como alguien dispuesto a establecer, en función de sus intereses, sus propios objetivos de aprendizaje, capaz de asumir su control tomando decisiones sobre qué, cómo, cuándo y dónde aprender y con quién (Coll, Engel, Saz y Bustos, 2014).

Con esta postura de aprendizaje de los PLE se crea la necesidad de tener una educación formal más abierta y flexible, que tenga en cuenta el aprendizaje realizado por el estudiante en los diferentes contextos y medios en los que se mueve y participe y promueva la articulación entre ellos. Hoy en día los estudiantes aprenden intercambiando información, compartiendo ideas a través de Facebook o twitter; realizando búsquedas en internet, grabando y subiendo videos o imágenes a YouTube; y creando presentaciones interactivas con Prezi, intercambiando archivos de audio con Audacity o Podcast, entre otras. Todas estas herramientas y recursos tecnológicos utilizados para aprender están a disposición tanto de docentes como de estudiantes y de cualquier persona que quiera utilizarlas; por lo tanto, lo que se debe hacer es buscar la metodología adecuada para reunirlas, sistematizarlas y hacerlas útiles para el desarrollo del aprendizaje de los estudiantes.

En relación con el diseño y la estructura de los PLE, y a partir de los postulados propuestos en este sentido por Adell y Castañeda (2013), se puede decir que estos presentan las siguientes características básicas: cada alumno se fija sus propios objetivos de aprendizaje, no hay evaluaciones ni títulos, no hay una estructura formal, internet pone a disposición de las personas un conjunto de herramientas y recursos gratuitos para compartir y aprender a través de ellos (véase la figura1).

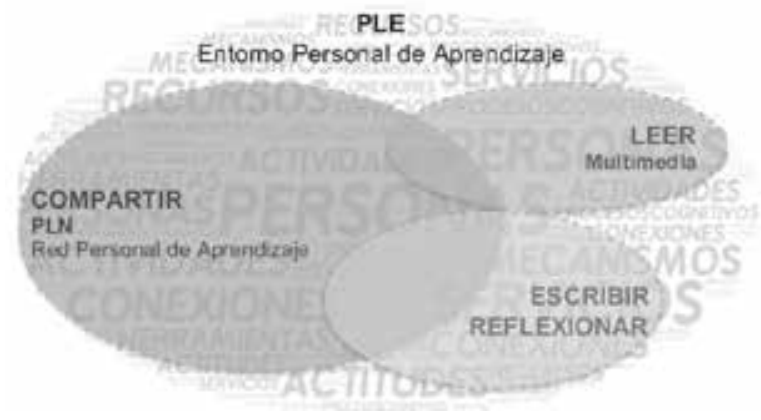

Figura 1. Componentes o partes de un PLE

Fuente: Adell y Castañeda (2013)

Las herramientas y recursos TIC que se pueden integrar en un PLE. Según Lara y Moraga (2013), son bastantes y diversos y es necesario hacer una selección de estos en función de las principales actividades, como: buscar y depurar información; organizar los contenidos mediante aplicaciones como Evernote, Springprad y otras; crear, editar y publicar información mediante la elaboración de blogs, wiki, google drive, zoo, mapas mentales o conceptuales; establecer comunicación con los demás, usando las redes sociales (Facebook, twitter, Tuenti, Skype, entre otras); compartir información y colaborar con otras personas en la creación de nuevos conocimientos, a través de las redes sociales, la creación de blogs (individuales, de aula), Glogster, Dropbox, 
Drive, entre otros; y organizar y gestionar el entorno personal mediante el empleo de herramientas tecnológicas como Symbaloo o Netbives, sitios para organizar un PLE.

Todos estos recursos se deben construir paso a paso, siguiendo una debida planificación determinada según los objetivos de aprendizaje que se plantean y los criterios que se establezcan para su implementación. Es necesario que los docentes diseñen actividades, tareas, proyectos de trabajo de aula que impliquen participación del estudiante y un uso intensivo de las herramientas de los PLE.

\section{Metodología}

Desde el marco metodológico, se implementa un diseño de investigación aplicada por medio de la investigación-acción-participación (IAP). Su implementación se desarrolló en cuatro fases comprendidas en 18 meses (de febrero de 2014 a noviembre de 2015), distribuidas en periodos de 4 a 5 meses, con una muestra poblacional de 25 estudiantes que cursaban los grados primero y segundo respectivamente. A partir de los momentos del diseño, las fases de la investigación se desarrollaron como se indica en la tabla 1 .

Tabla 1. Momentos del diseño

\begin{tabular}{|c|c|c|}
\hline $\begin{array}{c}\text { Interés técnico } \\
\text { Observar }\end{array}$ & $\begin{array}{c}\text { Interés práctico } \\
\text { Actuar }\end{array}$ & $\begin{array}{c}\text { Interés emancipatorio } \\
\text { Pensar }\end{array}$ \\
\hline Diagnóstico & Trabajo de campo & $\begin{array}{c}\text { Reflexiones: } \\
\text { ¿qué se transformó? }\end{array}$ \\
\hline $\begin{array}{l}\text { Se realizó la recolección de información } \\
\text { e ideas, planteamiento del problema } \\
\text { de investigación, búsqueda de } \\
\text { fuentes de información, rastreo } \\
\text { bibliográfico, fundamentación teórica } \\
\text { y estructuración del documento } \\
\text { del proyecto de investigación. } \\
\text { Posteriormente se diseñó el PLE y los } \\
\text { instrumentos de recolección de datos, que } \\
\text { luego de ser validados en la prueba piloto } \\
\text { pasaron a la fase de implementación }\end{array}$ & $\begin{array}{l}\text { La ejecución e implementación correspondió } \\
\text { a la tercera fase, donde se realizaron los } \\
\text { talleres y se ejecutó el cronograma de } \\
\text { actividades planteadas para los estudiantes, } \\
\text { iniciando con una etapa de producción de } \\
\text { materiales escritos, orales y audiovisuales } \\
\text { generados con herramientas y aplicaciones } \\
\text { de la web. Estos se compartieron en la } \\
\text { página web de la institución educativa, un } \\
\text { blog y la página web del proyecto, todas } \\
\text { conectadas y apoyadas con redes sociales. }\end{array}$ & $\begin{array}{l}\text { Al finalizar esta fase se realizó el } \\
\text { postest o cuestionario final, para } \\
\text { llegar a la última fase de análisis } \\
\text { y evaluación, en la que se realizó } \\
\text { el estudio de la información } \\
\text { recopilada en los momentos } \\
\text { y parámetros descritos en la } \\
\text { metodología de la investigación }\end{array}$ \\
\hline
\end{tabular}

Fuente: Jiménez B, Isabel. Investigadora Centro de Tecnologías de la Universidad de La Sabana

\section{Análisis de datos}

Los estudiantes fueron observados durante los talleres de implementación del ambiente y se tuvieron en cuenta los resultados de la rúbrica de valoración personal para registrar datos de las interacciones en la construcción del PLE. La información se consolidó de acuerdo a la categorización. Se realizó la codificación de las respuestas cualitativas de las encuestas realizadas a 25 estudiantes, y del registro de las observaciones, usando el programa de análisis
MAXQDA10. Se encontró información estadística generada por la codificación y segmentación, de acuerdo a la cantidad de segmentos asignados a una subcategoría y categoría, los cuales se discriminaron encontrando la frecuencia absoluta, en concordancia con la descripción de las categorías y los ítems de las rúbricas de valoración.

Para el análisis, se definen de manera deductiva e inductiva las categorías y subcategorías como se observa en la tabla 2. 
Universidad Pedagógica Nacional

Facultad de Humanidades

Tabla 2. Categorías de análisis de la investigación

\begin{tabular}{|c|c|c|}
\hline Categoría & Subcategoría & Descripción \\
\hline \multirow{3}{*}{ Componentes del PLE } & $\begin{array}{l}\text { Búsqueda y selección } \\
\text { de información }\end{array}$ & $\begin{array}{l}\text { La relación e interacción del estudiante con los } \\
\text { navegadores y buscadores, para acceder a la información, } \\
\text { las herramientas y programas que usa para clasificarla } \\
\text { y la habilidad que va adquiriendo para buscar y } \\
\text { encontrar información adecuada para sus intereses. }\end{array}$ \\
\hline & Producción y creación & $\begin{array}{l}\text { Desde los productos realizados por cada estudiante } \\
\text { y el proceso que llevó a cabo para lograrlo, hace } \\
\text { referencia a la parte del PLE del reflexionar y hacer, } \\
\text { con la información obtenida en la web. }\end{array}$ \\
\hline & Compartir y relacionarse & $\begin{array}{l}\text { El estudiante se relaciona, interactúa y se hace } \\
\text { visible en el mundo digital, a través de sus } \\
\text { publicaciones, realimentaciones a otros grupos } \\
\text { y redes de aprendizaje a los que se vincule. }\end{array}$ \\
\hline Categoria & Subcategoría & Descripción \\
\hline \multirow{2}{*}{ Procesos de aprendizaje } & Constructivismo & $\begin{array}{l}\text { Se observa el proceso de construcción de } \\
\text { conocimiento desde los intereses de cada estudiante, } \\
\text { sus conocimientos, la motivación para crear nuevos } \\
\text { y lo relacionado desde el constructivismo. }\end{array}$ \\
\hline & Aprendizaje autorregulado & $\begin{array}{l}\text { El estudiante logra involucrarse en el mundo digital } \\
\text { de manera autónoma, sigue instrucciones y regula } \\
\text { sus tiempos y espacios de trabajo, posterior } \\
\text { a la tutoría o instrucciones del maestro. }\end{array}$ \\
\hline \multirow{3}{*}{ Habilidades comunicativas } & Lectura & $\begin{array}{l}\text { El estudiante se hace un lector mas hábil } \\
\text { determinando el tema, el propósito comunicativo } \\
\text { y el tipo de texto, interpretando el lenguaje y } \\
\text { variaciones lingüisticas de cada tipo textual. }\end{array}$ \\
\hline & Escritura & $\begin{array}{l}\text { El estudiante logra producciones textuales en diferentes } \\
\text { formatos, con buena redacción, ortografía, cohesión y } \\
\text { coherencia a partir de un plan o un esquema de escritura, } \\
\text { relacionando sus conocimientos previos con nuevos. }\end{array}$ \\
\hline & Expresión oral & $\begin{array}{l}\text { Desde la expresión oral a partir de la narración, descripción, } \\
\text { declamación y otros, con el uso de vocabulario y } \\
\text { expresión acorde a diferentes momentos y situaciones. }\end{array}$ \\
\hline
\end{tabular}

Fuente: Elaboración propia

Diseño del ambiente de aprendizaje

En el diseño del ambiente de aprendizaje se tuvo en cuenta lo planteado por Sobrino sobre la autorregulación: "El éxito en la auto-regulación del aprendizaje es un compromiso entre la posibilidad del alumno de crear Entornos Personales de Aprendizaje y el ofrecimiento de estructura (scaffolding) por parte del docente" (2011, p. 131). Por consiguiente, este ambiente se construyó y desarrolló de manera conjunta basándose en los siguientes pasos:

- Diagnóstico y caracterización de la población

- Planificación de contenido, talleres y recursos

- Ejecución de talleres

- Realimentación y evaluación 
Así se diseña un modelo de ambiente de aprendizaje, denominado por los participantes como Cibernautastic-Aprendiendo en la web, basado en un diagnóstico de habilidades comunicativas y los datos estadísticos de caracterización de la población arrojados por la encuesta inicial, teniendo en cuenta la edad promedio, el nivel socioeconómico, los intereses de los estudiantes, saberes previos, habilidades, uso de herramientas y aplicaciones tecnológicas y disponibilidad de artefactos tecnológicos con conexión a internet de cada estudiante.

En este mismo orden, la metodología de trabajo del ambiente se basa en el constructivismo a partir del ejercicio de la planificación y ejecución de los talleres como estrategia didáctica $y$, por ende, la construcción e implementación del PLE de cada estudiante, como objetivo del proyecto de investigación. La evaluación es continua y permanente, desde los procesos enfocados al constructivismo, el trabajo colaborativo y la autonomía en el uso de las Tic, los intereses y necesidades de los estudiantes frente al uso de diversas herramientas y aplicaciones que les permitan estructurar su PLE, las condiciones espaciales, temporales y de infraestructura y las interacciones de los estudiantes en los PLE desde los tres componentes.

En cuanto a los recursos y materiales, se requiere el trabajo con artefactos tecnológicos como computadora, tableta, tablero inteligente, proyección con video beam y conexión a internet, para el trabajo desde plataformas o herramientas de comunicación sincrónica o asincrónica, específicamente con el uso de Google y sus aplicaciones. Al mismo tiempo, se facilita la combinación de diferentes materiales de tipo audiovisual, gráfico o textual desde la web, lo que hace un ambiente ecológico al reducir costos en impresiones o materiales de papelería.

El rol del estudiante es otro aspecto que debe tener presente el ritmo personal de aprendizaje, por lo que se proponen las actividades y ellos escogen las herramientas y contenidos relacionados, a la vez que siguen instrucciones de los maestros o tutores y aplican las enseñanzas para sus procesos de aprendizaje. Se presenta el trabajo colaborativo y desde lo individual son creadores y no consumidores que repiten información.
Es importante el rol del maestro en relación con el software educativo y los artefactos usados en un ambiente tecnológico. Algunos programas inciden en la creación de ambientes y situaciones de aprendizaje, donde el maestro puede ser reemplazado por el uso de programas, pero en otros entornos o ambientes será imprescindible su presencia, en consecuencia tendrá un nuevo rol, $y$ asumirá funciones de supervisor, facilitador y controlador de las actividades desarrolladas en el aula (Silva, 2010).

En la definición de tiempos y espacios, el aprendizaje virtual maneja horarios flexibles, que permiten trabajar desde cualquier lugar y no se limitan a la escuela, lo que amplía los escenarios de aprendizaje. Sin embargo, para organización del ambiente y desarrollo de los talleres, se dispuso del espacio del aula de informática 6 horas semanales en la jornada escolar, distribuidas en 3 sesiones de 2 horas, también en sesiones de 3 horas en contra jornada 2 veces a la semana.

\section{Resultados}

De acuerdo al análisis descriptivo se obtuvieron resultados desde la medición nominal con la determinación de las categorías, la clasificación, la selección, la cuantificación y cualificación de los datos, expresada en porcentajes, rangos o segmentos codificados que incluyen el uso de la medición ordinal en una escala valorativa determinada para las interacciones de los estudiantes en el PLE en los niveles de avanzado, satisfactorio, mínimo e insuficiente. La organización de datos cuantitativos se apoyó desde la estadística descriptiva con el programa Microsoft Excel y la de datos cualitativos con el procesamiento en el programa MAXQDA10.

El análisis de los datos obtenidos en cada instrumento se realiza de acuerdo a las tres categorías planteadas: Componentes del PLE, Procesos de aprendizaje y Habilidades comunicativas. Los datos de la encuesta pretest y postest, las valoraciones de las rúbricas y los formatos de observación se clasifican en primer lugar según sean de tipo cualitativo o cuantitativo. Los primeros se consolidan en el programa MAXQDA 10 para que se les asigne un código de acuerdo a las categorías preestablecidas, luego con los listados de los segmentos codificados se toma cada categoría y 
subcategoría, y de manera manual se determina la frecuencia absoluta por la repitencia o coincidencia de los datos encontrados, y se relacionan los segmentos. Los datos cuantitativos se organizan desde la estadística descriptiva, usando la generación de gráficos y tablas en las categorías y subcategorías.

Se presenta el consolidado de datos de la interacción inicial y después de la implementación en el PLE, según los datos del cuestionario, rúbricas de valoración y formatos de registro, a través de porcentajes, se describen por categorías y se relacionan los datos obtenidos en estos instrumentos, los cuales coinciden o son similares, lo que da coherencia a los hallazgos.

De esta manera, en la encuesta pretest se observan las interacciones iniciales de los estudiantes, datos previos a la implementación del ambiente y del PLE. En la categoría de componentes del PLE se observa que un $48 \%$ de la población no presentaba interacción con ninguno de los elementos ni reconocía el concepto e ignoraba cómo estructurarlo. Un $40 \%$ indicó que el acceso a internet y espacios de la web se usaba para ver las redes sociales de los padres u otros familiares o ingresar a páginas de juegos en línea, lo que muestra un uso como consumidores de información, y no para aprender o construir conocimientos. El 12\% de la población restante presentaba alguna interacción con los componentes del PLE sin reconocer aprendizaje con el uso de las TIC.

Durante la implementación se observó que los estudiantes necesitan leer para poder usar las herramientas web como navegadores y buscadores; aquellos que no estaban atentos a los mensajes de los programas o aplicaciones o que no sabían determinar el tipo de página por su dominio en la URL no encontraban la información tan fácilmente, o lo que hallaban era de mala calidad. En la subcategoría Leer, buscar y seleccionar la información, se determinó que $49 \%$ de los estudiantes alcanzaron un nivel avanzado, y $42 \%$, un nivel satisfactorio, al lograr desarrollar habilidades de búsqueda, las cuales implican el leer y seleccionar. Llegaron a este punto a partir de la necesidad de hallar información pertinente o acceder a información específica durante las actividades y talleres.

En la categoría Procesos de aprendizaje, con respecto a la pregunta “ ¿Cuánto pueden aprender en la tableta, computadora o celular con conexión a internet?", se observó un $60 \%$ en insuficiente, lo que indica una valoración en la que los estudiantes consideran inexistente el aprendizaje en la web o internet; $36 \%$ no reconocían que se puede aprender en la web, y manifestaron que generalmente en las clases de informática no usaban internet como apoyo pedagógico y en las casas no contaban con conexión; en caso de tenerla, faltaba asesoría de los adultos para generar procesos educativos. Estos datos indicaron que los niños, independientemente del interés y acceso al mundo digital o las herramientas de la web, no contaban con el ambiente adecuado para el aprendizaje.

En esta categoría se observó el proceso de construcción de conocimiento desde características del aprendizaje autorregulado y el constructivismo, en cuanto a cómo los estudiantes logran relacionar sus intereses y motivaciones personales para la construcción y el desarrollo de productos a través de actividades en los talleres, algunas veces con ejercicios establecidos para todos y otras con la opción de elegir de acuerdo a sus fines y necesidades.

Para la categoría Procesos de aprendizaje, desde el constructivismo, con indicadores como la motivación y los intereses de los estudiantes para aprender en el mundo digital, se involucra el aprendizaje autorregulado con el seguimiento de instrucciones y la autonomía para tomar decisiones y acciones. Se evidenció que inicialmente el interés y la motivación estaban presentes determinadas por el juego, pero no se reconocía el aprendizaje en la web. Con la implementación del proyecto desde el ambiente, se logró vincular el constructivismo con la autorregulación para que el estudiante genere procesos de aprendizaje desde la estructuración en su PLE. Así, en esta categoría se alcanzó el $36 \%$ en nivel avanzado y un $54 \%$ en nivel satisfactorio. Al final del proyecto, la población logró generar sus propios procesos y ritmos de aprendizaje, reconociendo que el PLE es la forma como aprende cada uno en el mundo digital.

En cuanto a Habilidades comunicativas, se preguntó a los estudiantes por el uso de la computadora, internet y herramientas tecnológicas para leer, escribir y expresarse. Se encontró que 11 \% lo hacían 
mucho, de manera regular un $25 \%$, en lo mínimo un $37 \%$, y un $27 \%$ no las usaba. Estos porcentajes indicaron que no reconocían la importancia de la lectura, escritura y oralidad en el mundo digital, y además no contaban en ese momento con las herramientas para desarrollar las habilidades comunicativas; realizaban procesos mecánicos para cada una de las habilidades y no con un sentido de aprendizaje.

En cuanto a la subcategoría Expresión oral, los participantes presentaron al inicio un nivel mínimo, con un $46 \%$, w insuficiente con un $32 \%$ pues no estaban relacionados con la oralidad como medio de comunicación y de expresión de sus ideas, emociones y sentimientos. En consecuencia, se hizo necesario que desde las actividades escolares se generaran acciones que lograran desarrollar o fortalecer esta habilidad. Los estudiantes se presentaban temerosos al hablar en público, la mayoría lo hacía con gritos y las palabras usadas generalmente no eran las adecuadas.

En el fortalecimiento de las habilidades de lectura, escritura y oralidad se muestra que los estudiantes necesitaron desarrollar esas habilidades para lograr la construcción de conocimiento y que, desde la implementación del ambiente, todos requirieron leer comprendiendo, escribir coherentemente y expresarse asertivamente, lo que se alcanzó, con la interacción continua y el desarrollo de actividades propicias para tales fines. De esta manera se logró que el $84 \%$ de los estudiantes reconocieran, utilizaran y desarrollaran las habilidades comunicativas, de acuerdo a procesos y espacios determinados por ellos mismos; el 31\% alcanzó el nivel avanzado, y el 53\%, satisfactorio.

Contrastando los porcentajes de interacción inicial y final en la implementación del PLE, de manera general, se observa en la figura 2 que los resultados obtenidos inicialmente se encontraban en niveles mínimos o insuficientes y que, con la implementación del PLE, pasaron a avanzados y satisfactorios. Las interacciones de los estudiantes en el PLE se encontraban inicialmente con un $5 \%$, para pasar al $42 \%$ en nivel avanzado, en el nivel satisfactorio se pasó del $12 \%$ al inicio al $46 \%$ al final, para un total de $88 \%$ de los estudiantes que lograron avances en su proceso de aprendizaje. El $12 \%$ restante está distribuido con el $9 \%$ en nivel mínimo de estudiantes a los que se les dificultaron los procesos, o requieren más tiempo para ellos, y el $3 \%$ de los estudiantes con dificultades en la implementación de su PLE por razones como ausentismo o desinterés.

Se indica entonces que la implementación del ambiente es adecuada, ya que propicia de manera integral el uso de TIC, recursos, herramientas y metodologías, con énfasis enla lectura, escritura y oralidad. Esto permite la continua construcción de esas formas de aprendizaje particulares desarrolladas a lo largo de la vida (véase la figura 2).

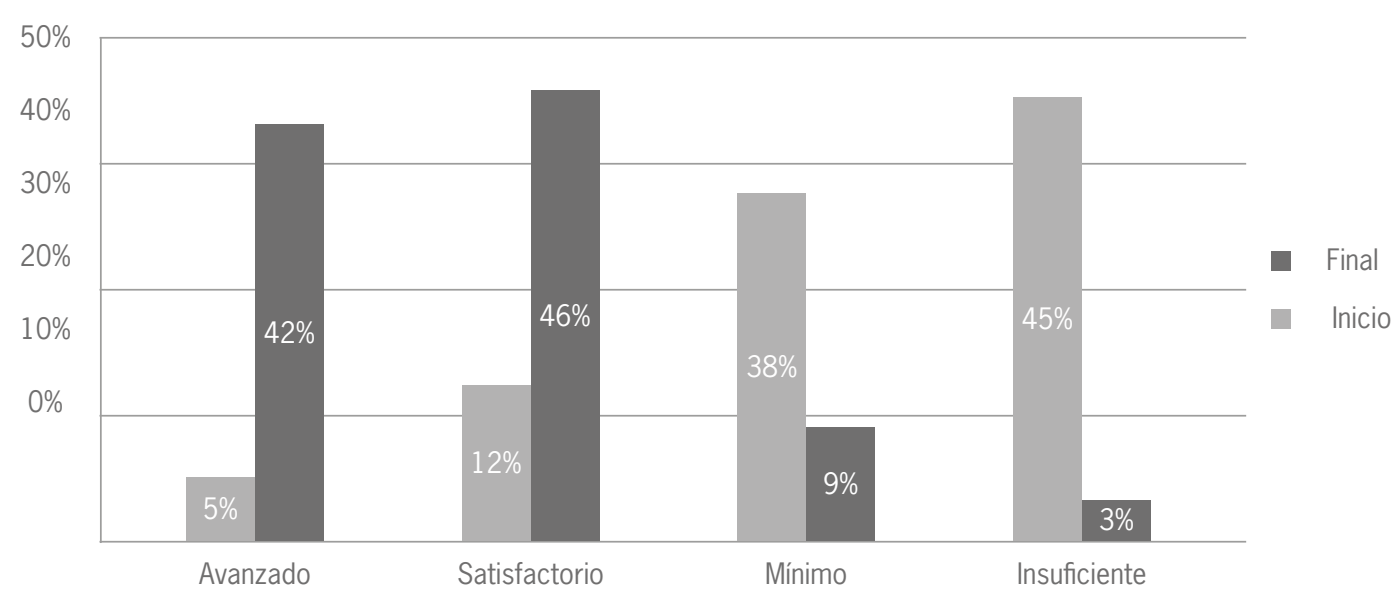

Figura 2. Interacciones generales en el PLE al inicio y al final. 


\section{Discusión}

Para dar respuesta a la pregunta de investigación “Cómo la implementación de entornos personales de aprendizaje a través de un ambiente de enseñanza y aprendizaje, logra fortalecer las habilidades comunicativas en Básica Primaria?”, se concluye que:

La implementación de los PLE debe realizarse desde un ambiente basado en el constructivismo y el aprendizaje autorregulado, que involucre el desarrollo de sus tres componentes, donde las habilidades comunicativas están presentes permanentemente con la necesidad del leer comprendiendo, escribir de manera coherente, escuchar como parte del seguimiento de instrucciones y el hablar para expresar sus ideas a través de productos que evidencien su aprendizaje.

Se requiere el diseño de un ambiente basado en brindar a los estudiantes experiencias de socialización de saberes con sus pares y maestro, el cual permite la toma de decisiones conjunta, el desarrollo de su pensamiento crítico y autonomía para la realización de sus actividades. Allí el estudiante reconoce que puede aprender sin imposición, lo que estimula su aprendizaje por medio de la exploración que permite el uso del PLE y promueve el desarrollo de habilidades comunicativas gracias a la práctica continua dentro del ambiente.

Frente al proceso de evaluación del aprendizaje, este se pudo monitorear a partir del desarrollo de actividades más allá de la calificación dada por el maestro. Se observó que el propio PLE estimuló en los estudiantes el deseo de avanzar a partir de los retos que este espacio les posibilitaba; además, en procesos de contrastación con sus pares y maestros, se pudo identificar la cualificación de su propio aprendizaje. Esto generó una coevaluación y autoevaluación permanente que brindó un clima de trabajo enmarcado en la confianza, el respeto y la valoración de los avances de aprendizaje particulares.

Con respecto a los obstáculos o categorías emergentes, estos originan en uno de los primeros componentes del PLE, cuyo objetivo es utilizar la información encontrada en la web para identificar sus saberes previos. Esta no es una tarea fácil, debido a factores como el que las instituciones educativas tengan varias restricciones en relación con la navegación, y al papel que debe desempeñar el maestro frente a la orientación de esta búsqueda ya que sus representaciones sociales sobre un tema específico pueden terminar sesgando el interés de los niños en estas edades iniciales.

Este argumento plantea perspectivas en cuanto a la tarea que tiene la escuela de seguir trabajando en temas relacionados con la prevención, para reconocer cómo ser un buen ciudadano digital, capaz de conocer y ejercer sus derechos y deberes ciudadanos, practicarlos y respetarlos en todos los aspectos de su vida. Esto implica que los utilice con coherencia tanto en su vida diaria, como cuando haga uso de las TIC.

En el caso de esta experiencia, dicho aprendizaje aportaría a las competencias de selección crítica que deben tener los niños para trabajar con los PLE. No obstante, en los resultados de esta investigación, los estudiantes adquirieron habilidades y autonomía en el uso de herramientas de la web e interacción en el mundo digital, lograron crear productos visibles a los demás, desde lo audiovisual, textual y gráfico, donde cada uno reguló sus tiempos y su compromiso, sin establecer que eran actividades para entregar, sino creaciones por desarrollar.

Desde la categoría Componentes del PLE, se encontraron aspectos importantes para tener en cuenta, como el desarrollo de habilidades de búsqueda y selección, lo cual es difícil para niños de corta edad; con la cantidad de información que está en la internet, es un reconocimiento el manejo que les dieron a los datos encontrados. También lograron conformar sus espacios virtuales, a través de blogs, páginas web, canales de YouTube, generando motivación y satisfacción personal, pues estos entornos van más allá de compartir fotos para compartir aprendizajes, y lograr otra mirada de la web.

En los procesos de aprendizaje, son importantes los saberes y experiencias previas para activar esquemas de conocimiento y así desarrollar nuevos esquemas y significados, cambiando al pensarse el porqué, cómo y para qué de la información obtenida. En cuanto al uso de las habilidades de lectura, escritura, escucha y 
oralidad se logró que los estudiantes reconocieran la importancia de trabajar en esto continuamente, que es algo para su vida pero que impacta en la de los demás. Lograron expresarse mejor, darse a conocer y generaron la necesidad del desarrollo de actividades para fortalecer sus habilidades comunicativas.

Finalmente, los aspectos relevantes que ofrece la experiencia pedagógica de usar PLE para jalonar los procesos de aprendizaje pueden resumirse en: experiencias de innovación con el uso de tecnologías para la enseñanza así como para el aprendizaje en los niños, el desarrollo de habilidades del siglo XXI tales como el desarrollo de competencias digitales para reconocerlas e implementarlas en su experiencias cotidianas, el desarrollo del pensamiento crítico al ser selectivo en la información que encuentran en la web para uso académico o de entretenimiento, las competencias como el trabajo colaborativo como parte de la formación social y política al ser capaz de reconocer al otro con roles iguales y roles diferentes con saberes previos y capacidades para construir en colectividad.

\section{Referencias}

Adell, J. y Castañeda, L. (2013). Entornos personales de aprendizaje: claves para el sistema educativo en red. Alcoy: Marfil.

Ampudia, V. y Trinidad, L. (2012). Entornos personales de aprendizaje ¿final o futuro de los EVA? Reencuentro, 63, 32-39.

Bohada, L. (2016). Ambiente de aprendizaje mediado por software social para favorecer la apropiación de conceptos básicos de robótica. Recuperado de http:// intellectum.unisabana.edu.co/handle/10818/26523

Cabero, J. (2013). El aprendizaje autorregulado como marco teórico para la aplicación educativa de las comunidades virtuales y los entornos personales de aprendizaje. Teoría de la Educación: educación y cultura en la sociedad de la información, 14 (2), 133-156.

Castañeda, L., y Soto, J. (2010). Building Personal Learning Environments by Using and Mixing ICT Tools in a Professional Way. Digital Education Review, 18, 9-25.

Coll, C. y Engel, A. (2014). Introducción: los Entornos Personales de Aprendizaje en contextos de educación formal. Cultura y Educación, 26 (4), 617-630.
Gil, M. (2012). Desarrollo de entornos personales de Aprendizaje (PLES) para la mejora de la competencia digital. Estudio de caso en una escuela Italiana. Burgos, España: Universidad de Burgos.

Hernández Fernández M. A. (2016). Gestión del conocimiento, actividad científica y entornos personales de aprendizaje (PLES): una bibliometría de la PLE Conference. Edutec, Revista Electrónica Tecnología Educativa, 55, 1-16.

Lara, J. y Moraga, C. (2013). Diseño e implementación de entornos personales de aprendizaje en secundaria. e-CO. Recuperado de: http://www.revistaeco.cepcordoba.org/

Llorente, C. (2012). El e-learning 2.0. De la Tecnología a la metodología. Revista d'Innovacio Educativa, 8, 79-86.

López, L. (2015). Entornos personales de aprendizaje -PLE-: una mediación con TIC. (Pág. 1-33). Universidad de La Sabana.

Marín-Juarros, V., Negre-Bennasar, F. y Pérez-Garcias, A. (2014). Entornos y redes personales de aprendizaje (PLE-PLN) para el aprendizaje colaborativo. Comunicar, 42.

Ministerio de Educación Nacional. (2008). Orientaciones generales para la educación en tecnología. Ser competente en tecnología: Una necesidad para el desarrollo. Serie Guías, 30. Proyecto Ministerio de Educación Nacional (MEN) - Ascofade. Bogotá: Imprenta Nacional.

Monsalve, M. E., Franco, M. A., Monsalve, M. A., Betancur, V. L., y Ramírez, D. A. (2011). Desarrollo de las habilidades comunicativas en la escuela nueva. Educación y Pedagogía, 21(55), 189-210. Recuperado de http://aprendeenlinea.udea.edu.co/revistas/index. php/revistaeyp/article/viewArticle/9766

Navas, E. E. (2013). Los entornos personales de aprendizaje en el marco de la educación permanente. Edmetic, 2(1), 94-110.

Noguera, I., García, I., y Gros, B. (2014). Just4me: diseño pedagógico y funcional de un PLE para la autogestión del aprendizaje en distintos contextos. Cultura $y$ Educación.

Restrepo, A. (2016) Construcción de un entorno personal de aprendizaje con el propósito de fortalecer los niveles de lectura de los estudiantes de séptimo grado del Colegio Almirante Padilla. Recuperado de http:// intellectum.unisabana.edu.co/handle/10818/26028

Rodríguez, G. M. (2013). Una experiencia de creación de un entorno personal de Aprendizaje. Proyecto Dipro 2.0. Revista de Educación Mediática y TIC, 2(1), 7-22. 
Ruiz-Palmero, J., Sánchez Rodríguez, J., y Gómez García, M. (2013). Entornos personales de aprendizaje: estado de la situación en la Facultad de Ciencias de la Educación de la Universidad de Málaga. Pixel-Bit. Revista de medios y Educación, 42, 171-181.

Santamaría, F. (2010). Evolución y desarrollo de un entorno personal de aprendizaje en la Universidad de León. Revista Digital Education Review, 48-60. Recuperado de: http://www.greav.ub.edu/der/

Silva, S. S. (2010). Informática educativa. Uso y aplicaciones de las nuevas tecnologías en el aula. Bogotá:
Ediciones de la U. Pontevedra, España: Ideas Propias Editorial.

Sobrino, M. A. (2011). Las tecnologías de la información y de la comunicación (TIC) y los nuevos contextos de aprendizaje. Revista semestral del departamento de educación facultad de filosofía y letras, 20. Recuperado de: http://dadun.unav.edu/bitstream/10171/18344/2/ ESE\%20117-139.pd

The PLE Conference 2010. (s. f.). Recuperado de http:// pleconference.citilab.eu/ 